\title{
A HIERARCHICAL RULE-BASED METHOD FOR IMAGE SEGMENTATION USING MAXIMUM GRADIENT PROFILES
}

\author{
A.C.F.Colchester ${ }^{1}$ \\ R. T. Ritchings ${ }^{2}$ \\ N.D.Kodikara
}

1 Department of Neurology, Guy's Hospital, London Bridge SEl 9RT
2 Departinent of Computation, UMIST, PO Box 88 , Manchester M60 lQD

For each pixel in an image we determined the local direction of greatest grey level change and linked pixels in this direction to form maximum gradient profiles (MGP). Adjacent MGP's were linked side-by-side to form edge sections. These edge sections corresponded very closely to those from a Canny operator.

For the present application, which was concerned with detection and characterisation of blood vessels on angiograms, subsequent grouping was restricted to anti-symmetric edge sections which formed a ridge with high grey values in the centre. The ridges corresponded closely with blood vessels shown in the image. The width of each ridge and the locus of its centreline were derived from antisymmetric MG $P$ a i r s and corresponded very well with expert judgement of vessel width and centre line location. Advantages of the MGP grouping method included explicit representation of the profiles of grey values normal to edges; ease of implementation of higher-order grouping stages which were natural extensions of lower level processes; and hierarchical data structure representing image features at all scales, allowing improved interaction of bottom-up and top-down processing.

\section{Introduction}

our previous work on knowledge based interpretation of angiographic images $1,2,3$ has underlined the need to establish a hierarchical data structure which represents the image at multiple scales and which allows simplified control of the interaction between image-driven and knowledge-driven processes. The method has been developed as a potential alternative to the current multiresolution techniques which use image blurring of different degrees in the analysis of features at multiple scales 4 . We have developed the method in the context of the analysis of blood vessel images but it is not restricted to angiograms and, as will be seen, very few of the processing steps use domainspecific knowledge.

We consider an image as a 3-D grey level landscape with topological features ${ }^{5}$ and we treat an edge as a "hillside" or steep region on this 3-D surface. The most fundamental component of the edge is the profile of grey level change along lines of maximum gradient. Adjacent lines of maximum gradient on an edge will have similar profiles and tend to be approximately parallel. The directions of these maximum gradient profiles (MGP's) are normal to the direction of the edge. Our method of extracting image structure is based on MGP's, is easy to implement, and has d number of important properties. 


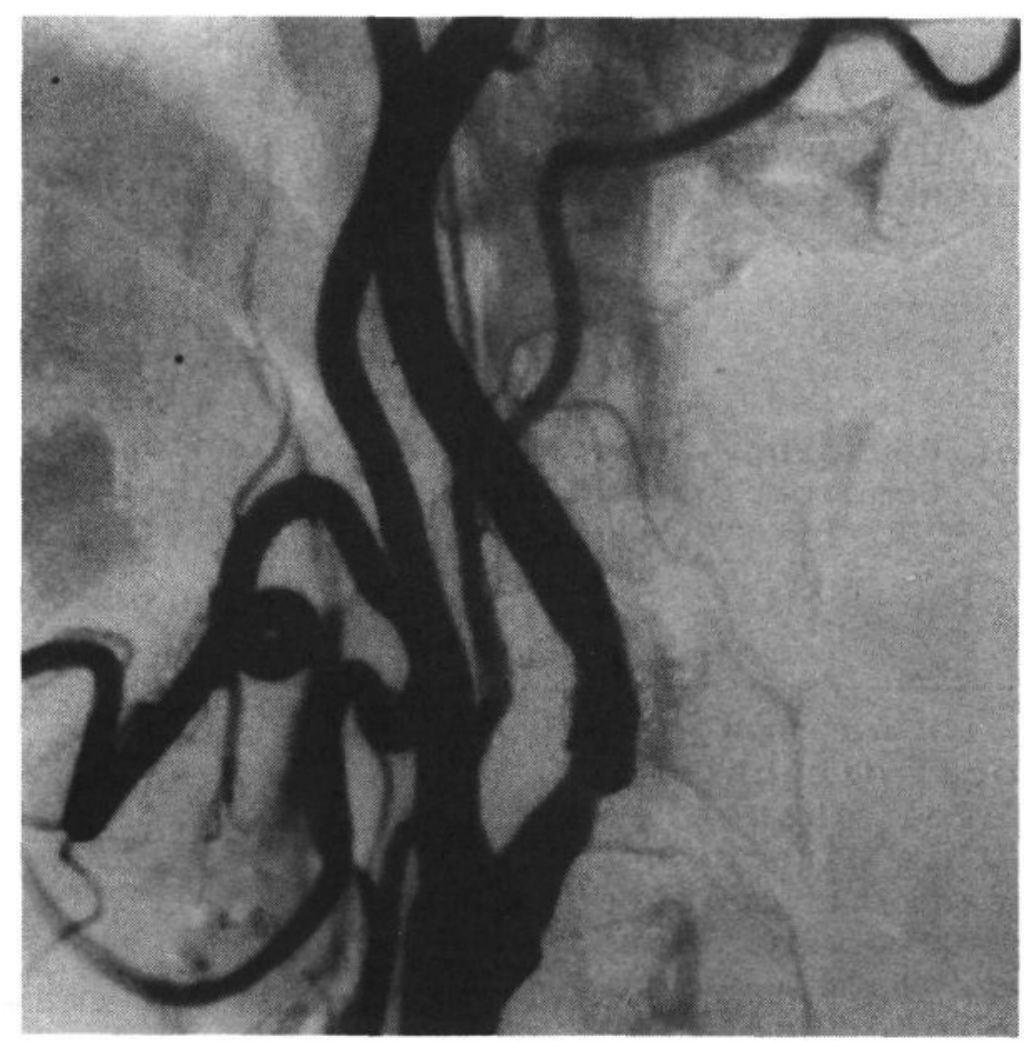

Figure 1 Region of interest before digitisation

\section{Method}

Conventional angiographic images showing the carotid arteries and their branches in the neck were analysed. They had already been photographically subtracted i.e. radiographic film obtained before injection of contrast medium had been subtracted from a film taken immediately after injection. Regions of interest on the angiograms were digitised to $128 \times 128 \times 6$ bit resolution. Examples are shown in Figs 1 and 8. The images were transferred to an Apollo DN 3000 workstation in the Departinent of Computation at UMIST where the system was implemented.

A pair of $3 \times 3$ sobel operators was used to establish the local direction and magnitude of maximum gradient for every pixel. A threshold for these gradients was derived automatically as follows. The image was divided into nonoverlapping $10 \times 10$ pixel squares and the sobel operator magnitudes (regardless of direction) were examined within every square. The square with the minimum standar'd deviation of Sobel magnitudes('s') was used for calculating the threshold which was then applied globally. All pixels whose Sobel output was greater than $3 \mathrm{~s}$ were retained. The neighbours of each of these pixels were examined, and any whose sobel gradient magnitude was greater than $2 \mathrm{~s}$ were also retained. other pixels were iynored.

Subsequent processing steps involved successively grouping elements to form larger elements, larger elements into still larger elements, and so on. Pixels with a local gradient above the threshold were linked along the path of maximum gradient to form MGP's provided that the track of an MGP did not change direction by more than $45^{\circ}$ in total. MGP's were terminated if there were no gradients above threshold or if a further link would involve a change of direction of more than $45^{\circ}$. The overall direction (in $x$ \& y) of the MGP was obtained from a least squares straight line fit to the $(x, y)$ value of the MGP. At 
the ends of each MGP neighbouring MGP's with the nearest end points were examined, and if the MGP's were approximately co-linear they were linked end to end to form a composite MGP. The point(s) of steepest slope of the MGP were found. MGP's whose steepest slope points were near neighbours were compared, and where these MGP's had similar direction and maximum gradient they were grouped to form a side by side set of MGP's or "hillside" region with a normal given by the mean of the MGP directions. Edge sections were formed from the points of steepest slope on adjacent MGP's by means of a rule set which (a) attempted to maintain the edge section direction perpendicular to the MGP's and (b) minimised small deviations in the direction of the edge section (similar to a 5-point smoothing). Where appropriate the rule set chose between competing steepest-slope points on a single MGP or interpolated new points. The edge sections thus derived were compared with edge sections derived frorn Canny and from MarrHildreth operators. The scale parameter of these was set at 11 pixels after some experimentation.

Processing using the edge sections derived from the MGP grouping method was continued by linking near-neighbour anti-symmetric edge sections to form ridges or valleys. If these were long in extent they corresponded to line features in the image. In the present application, where the objective was to detect blood vessels on good quality angiographic images on which blood vessels appeared as ridyes on the 3-D surface, edges without antisymmetric partners forming a ridge were suppressed. Along the length of ridges the width of the ridge and the locus of its centre-line or symmetric axis were derived from the edge points of antisymmetric MGP pairs.

The majority of the code was in the form of conditional rules programmed in Lisp; the sobel operator was programmed in Pascal.

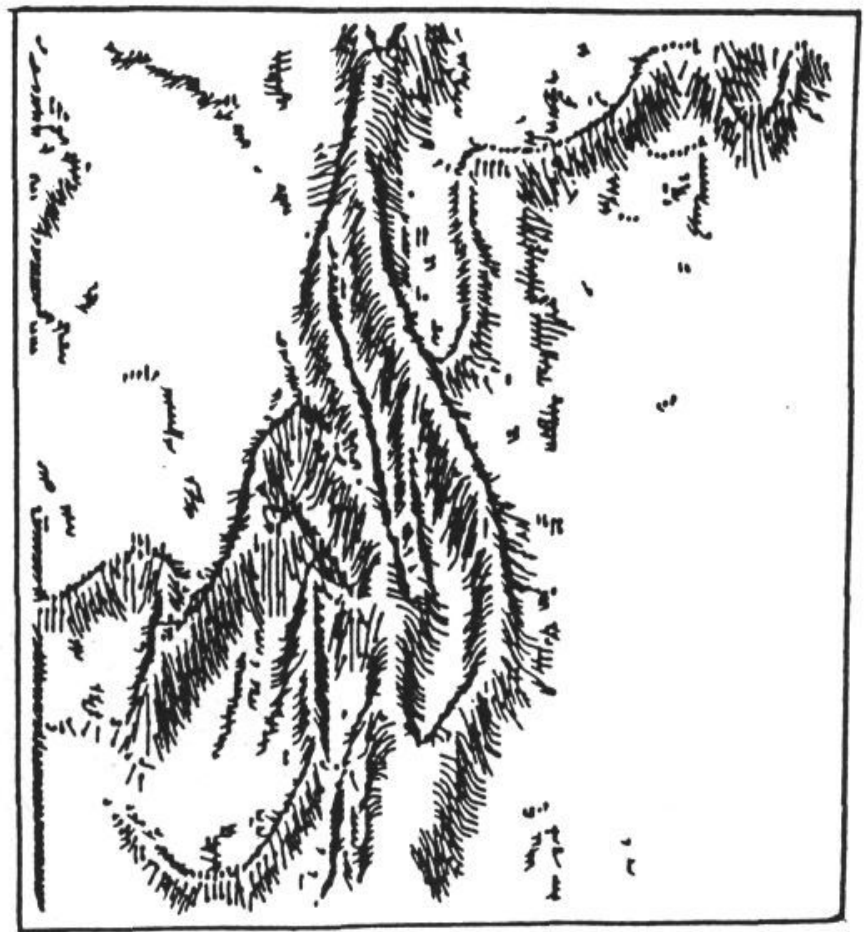

Figure 2A Angio 1: Perspective view of Maximum-Gradient Profiles shown as if superimposed on the 3-D grey level surface

\section{Results}

Two examples of the digitised region of interest are shown in Figures 1 and 8 . Figs 2 to 5 show stages of processing of Angiogram l, Figs 9 to 12 stages of Angiogram 2. Perspective views of the extracted MGP's, shown as if superimposed on the 3-D grey level surface of the images, are shown in Figures 2 and $9 . \quad F i g ~ 2 B$ is an enlarged view of a region just above the centre of Fig 2A. When the edge points are displayed the method can be seen to function as a very effective low level edge detector (Figures 3 and 10 ). With further processing to extend edge sections and remove those sections without an anti-symmetric partner forming a ridge, a reliable blood vessel boundary map was generated (Figures 4 and 11 ). The detected vessel centre lines, which corresponded very well with an expert's judgement of centre line position, are shown in Figures 5 and 12 . 


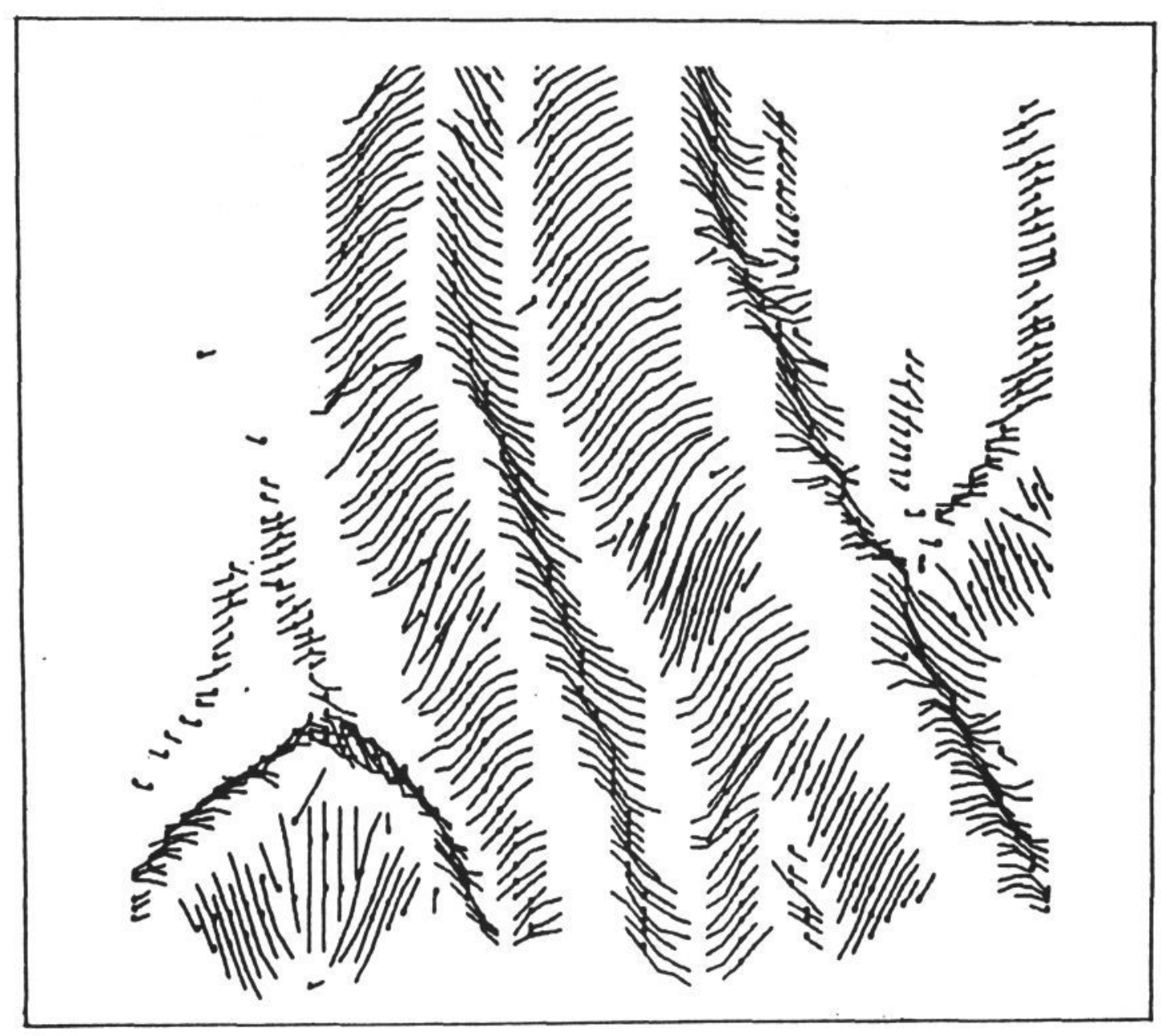

Figure 2B Angio 1: Enlarged view of Maximum Gradient Profiles from region near centre of Figure 2A 
Edge sections detected by the MGP grouping method (Figs. 3 and 10 ) were compared with the Canny (Figs 6 and 13) and Marr-Hildreth (Figs 7 and 14) operators. The MarrHildreth method suffers from major distortion at sharp corners. The MGP grouping method performs comparably to the Canny (compare Figs 3 with 6 and 10 with 13).

\section{Discussion}

Conventional image pre-processing typically involves the convolution of simple kernels with image grey data and then thresholding to generate edge sections. These then

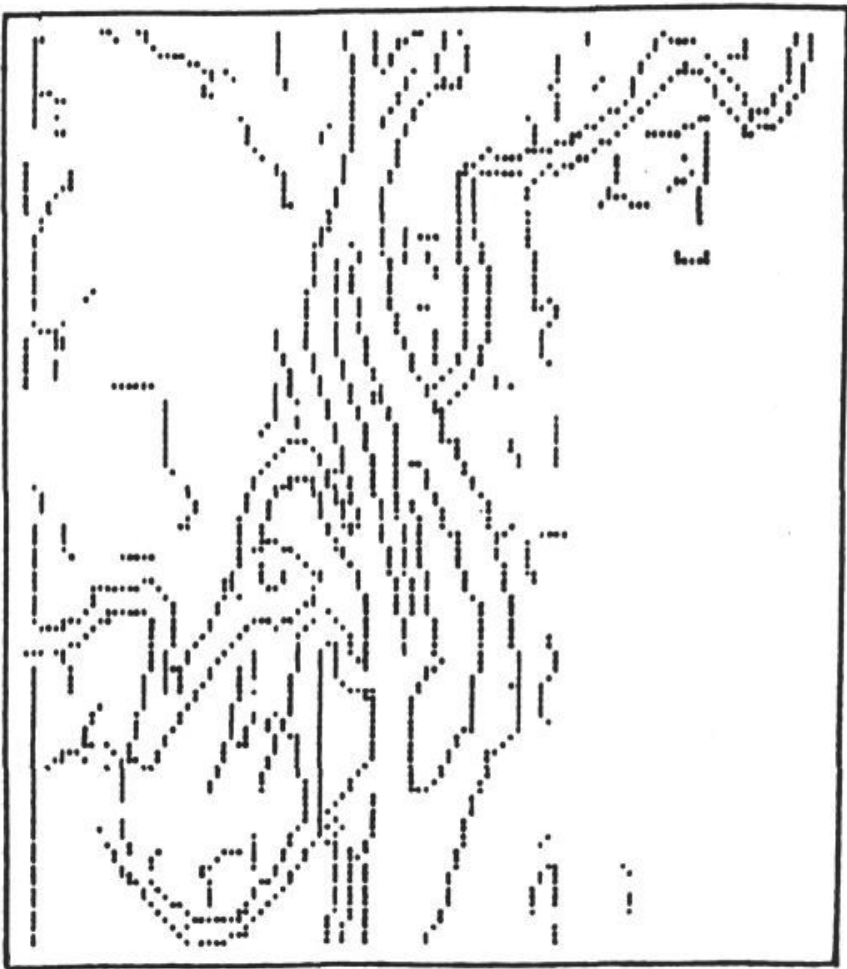

Figure 3 Angio 1: steepest-slope points on the Maximum Gradient Profiles

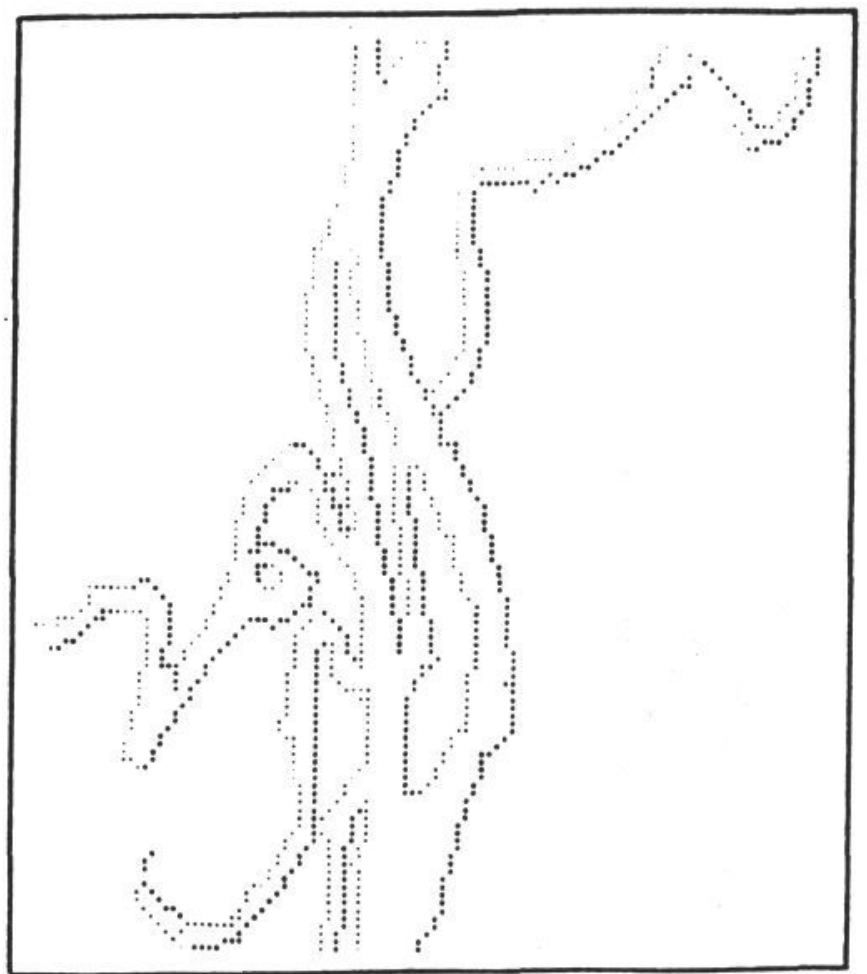

Figure 4 Angio 1: Blood vessel boundary map derived from MGP grouping method

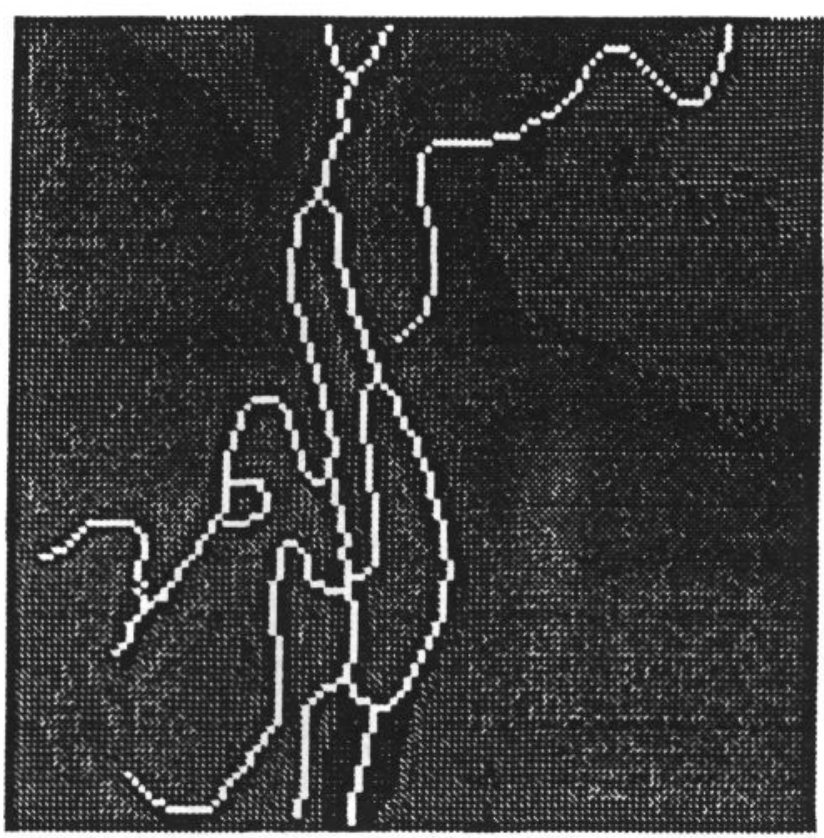

Figure 5 Angio 1: Blood vessel centrelines derived from MGP grouping method 


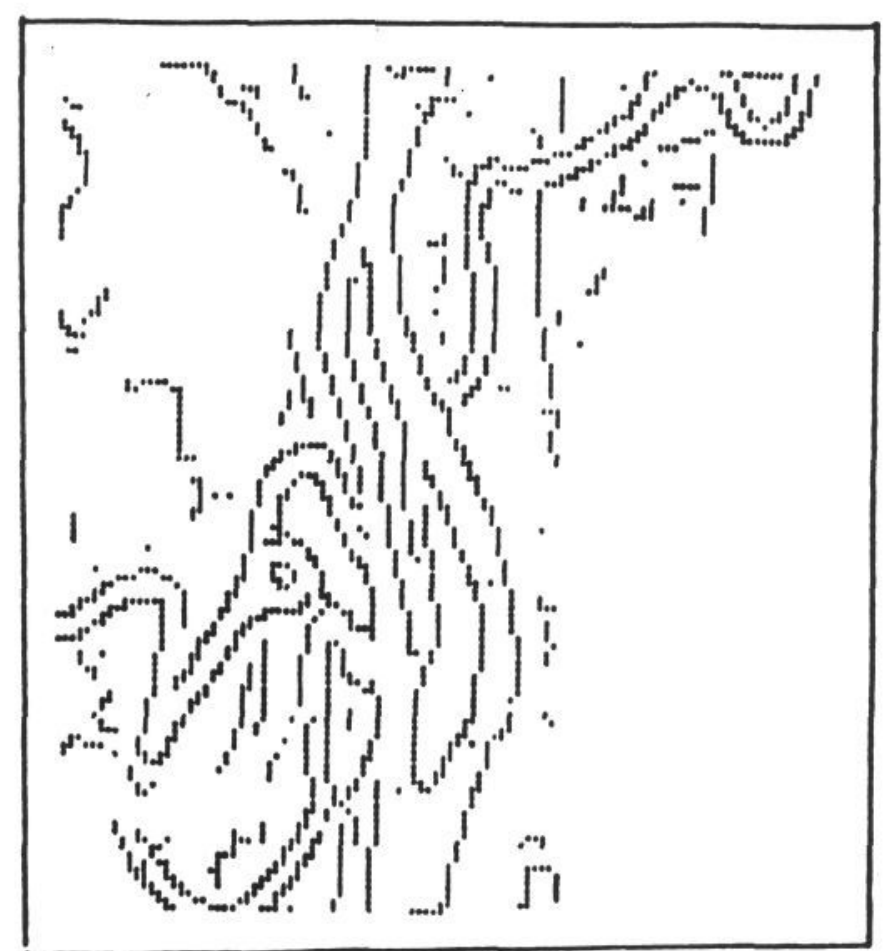

Figure 6 Angio 1: Canny operator output with width $=11$ pixels

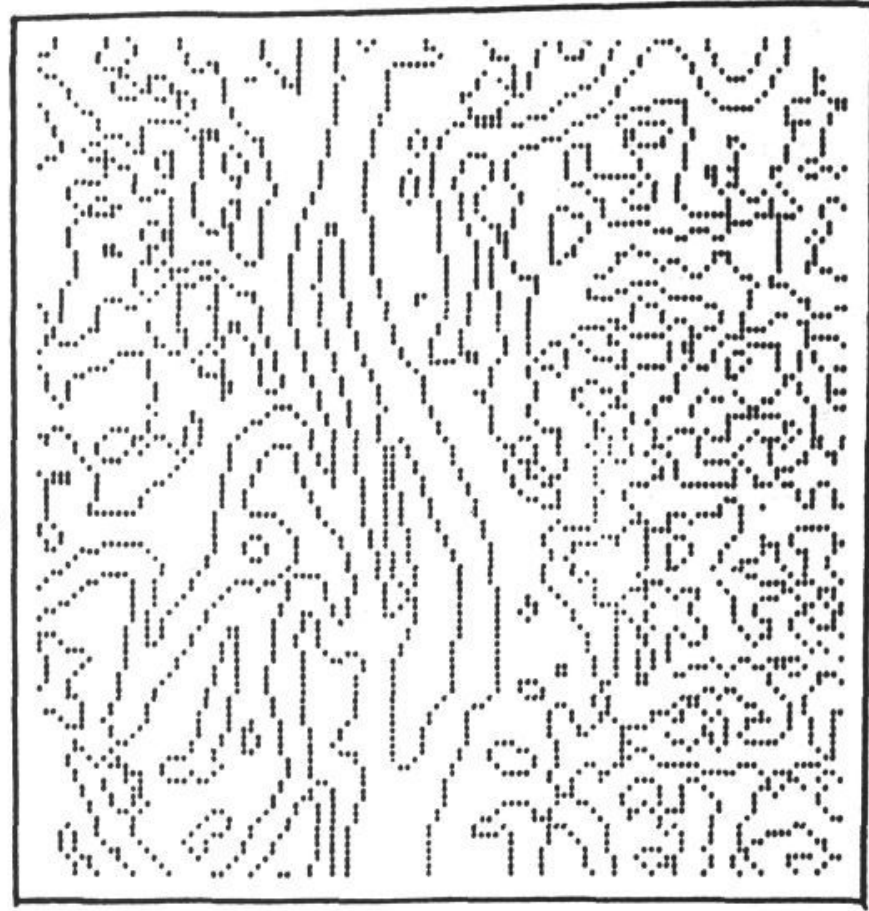

Figure 7 Angio 1: Marr-Hildreth operator output with width $=11$ pixels

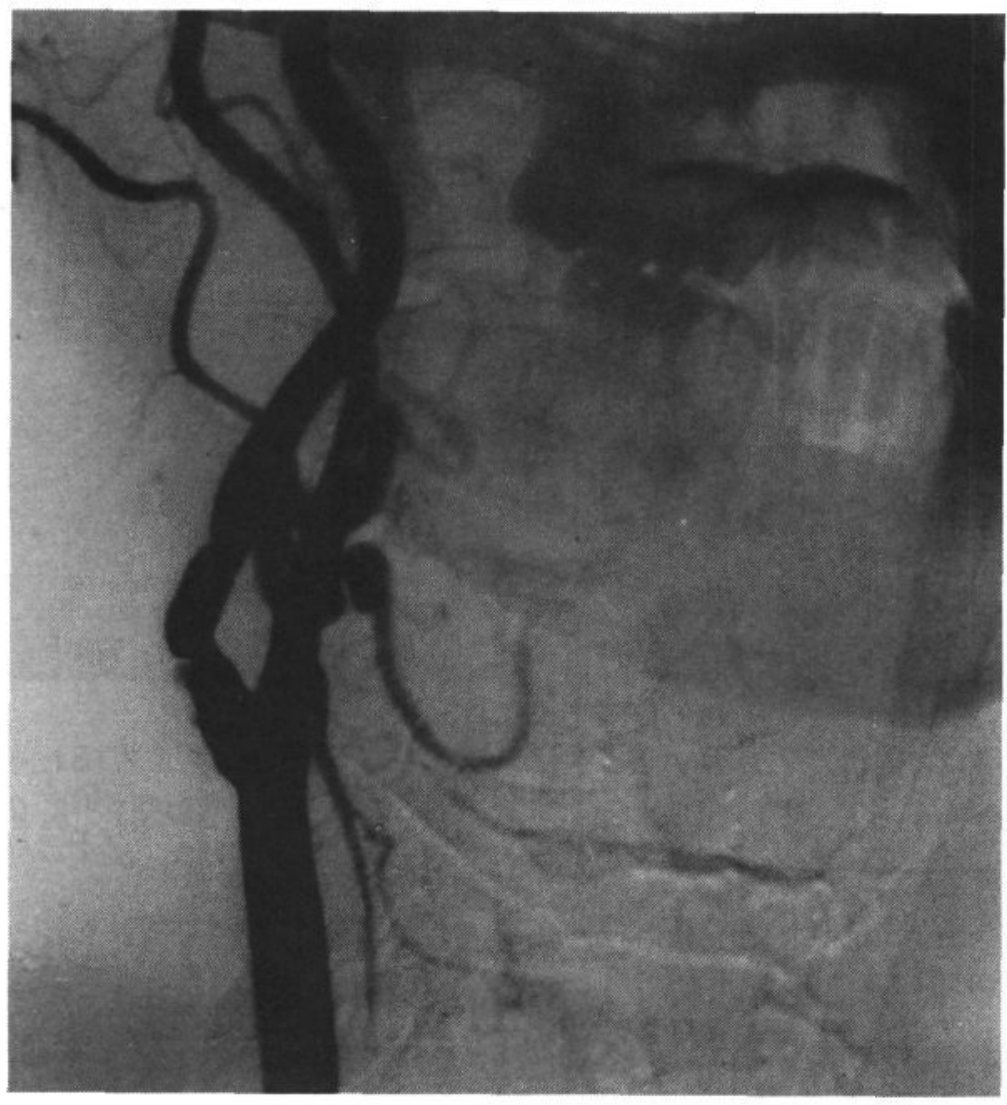

Figure 8 Angio 2: Region of interest before digitisation 


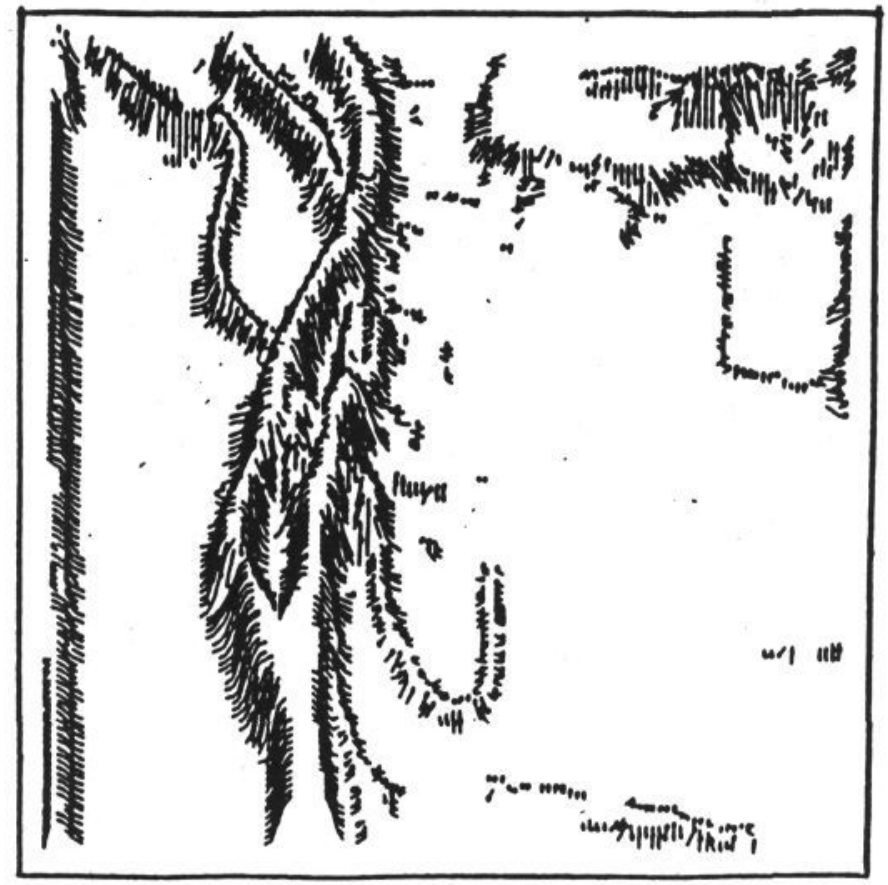

Figure 9 Angio 2: Perspective view of Maximum-Gradient Profiles shown as if superimposed on the 3-D grey level surface

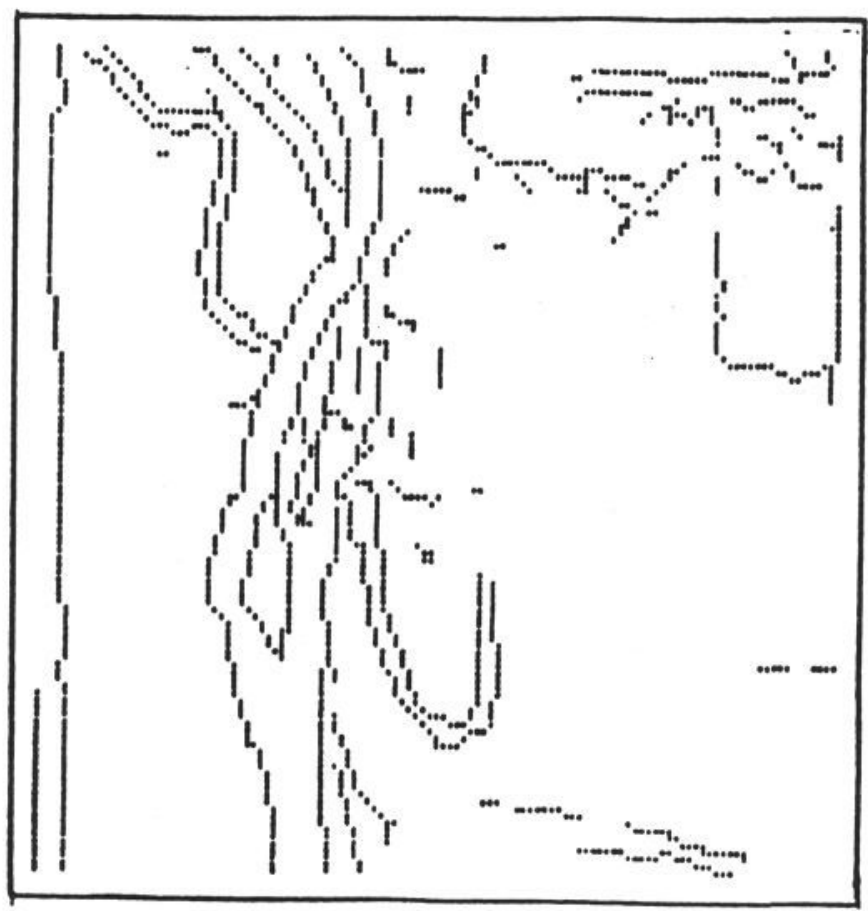

Figure 10 Angio 2: Steepest-slope points of the Maximum Gradient Profiles

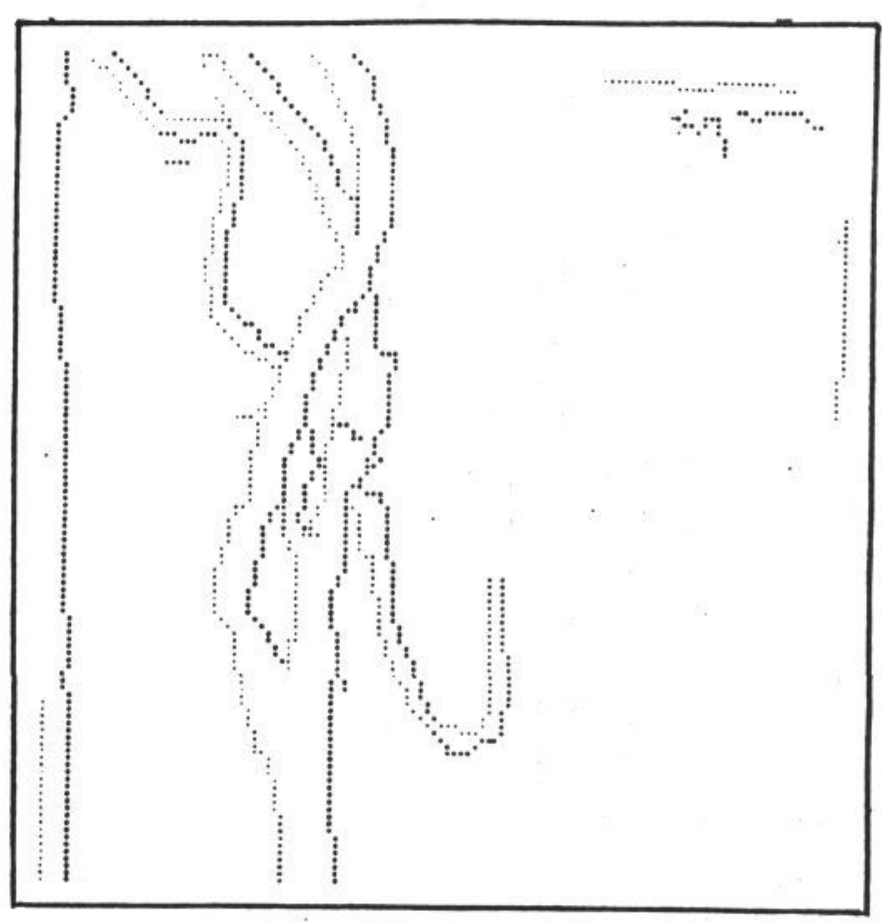

Figure 11 Angio 2: Blood vessel boundary map derived from MGP grouping method

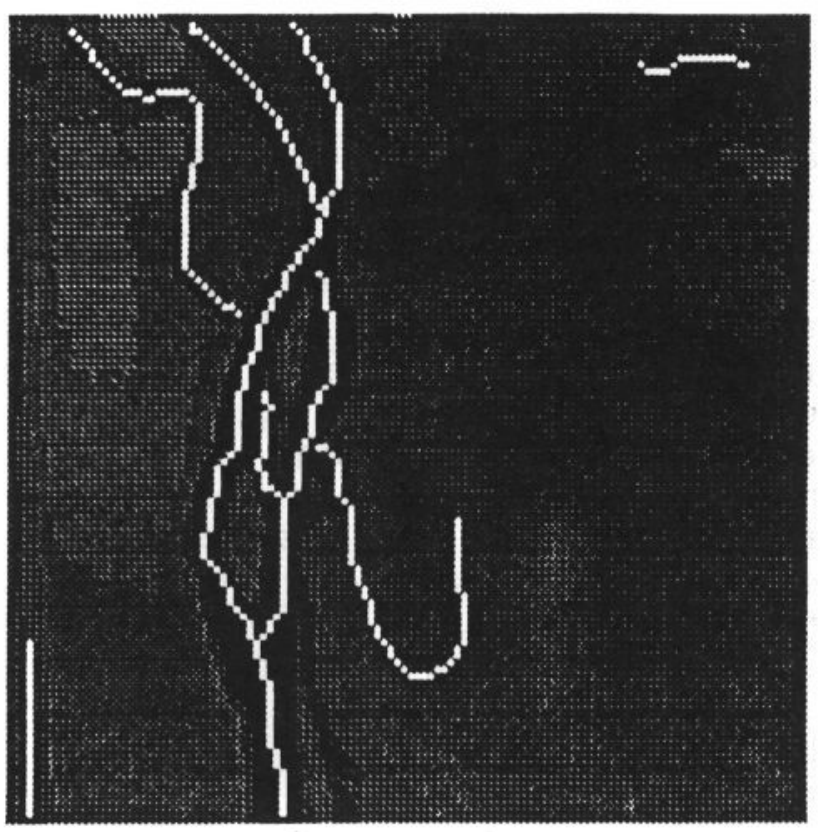

Figure 12 Angio 2: Blood vessel centre lines derived from MGP grouping method 


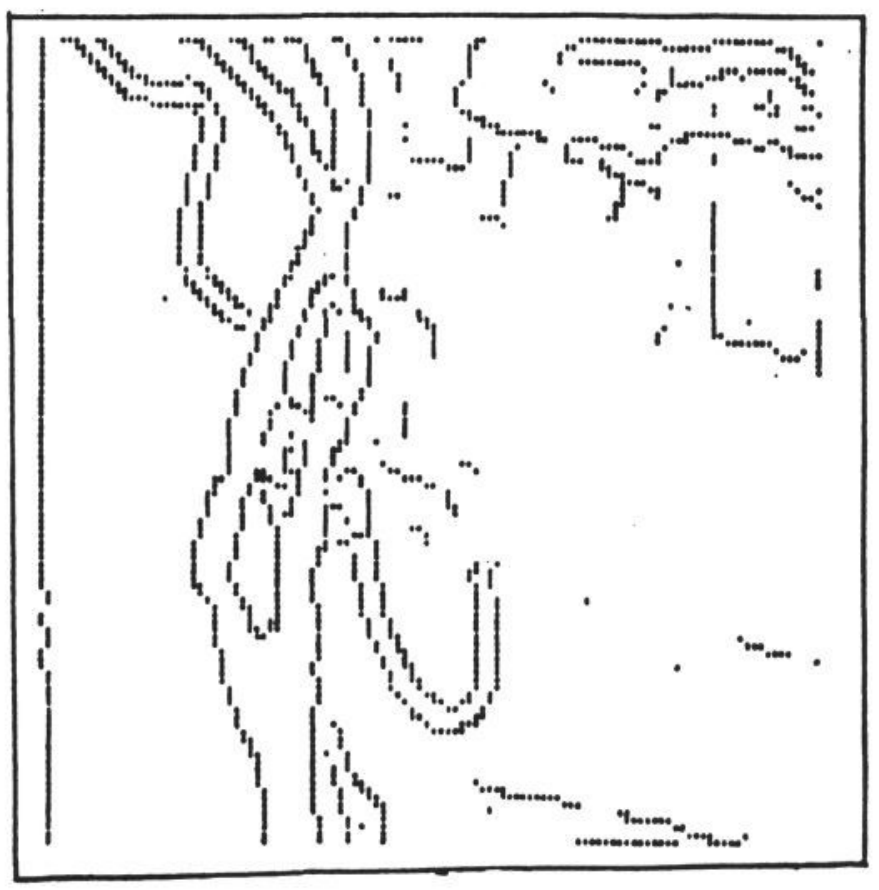

Figure 13 Angio 2: Canny operator output with width $=11$ pixels

have to be subjected to a different type of processing ${ }^{6}$ for linking, skeletonisation, etc. Our approach also requires the same type of geometric linking processes to group and extend edge sections into larger edge sections, larger edge sections into objects etc., but these same processes are also used at lower levels to generate edge sections. The distinction between preprocessing and subsequent analysis is thereby blurred. Similarly, the same basic data structures which are used for high-level, more abstract representations of objects can be used throughout the processing routine. In these ways, feedback to refine earlier processing results can be controlled in a more uniform manner and less re-processing of image grey-level data is required.

With our method, each stage of processing groups smaller elements into larger features. In that the same basic grouping rules are applied at each stage, and the number of features progressively decreases, our method is a type of pyramidal algorithm 7,8 with rapid

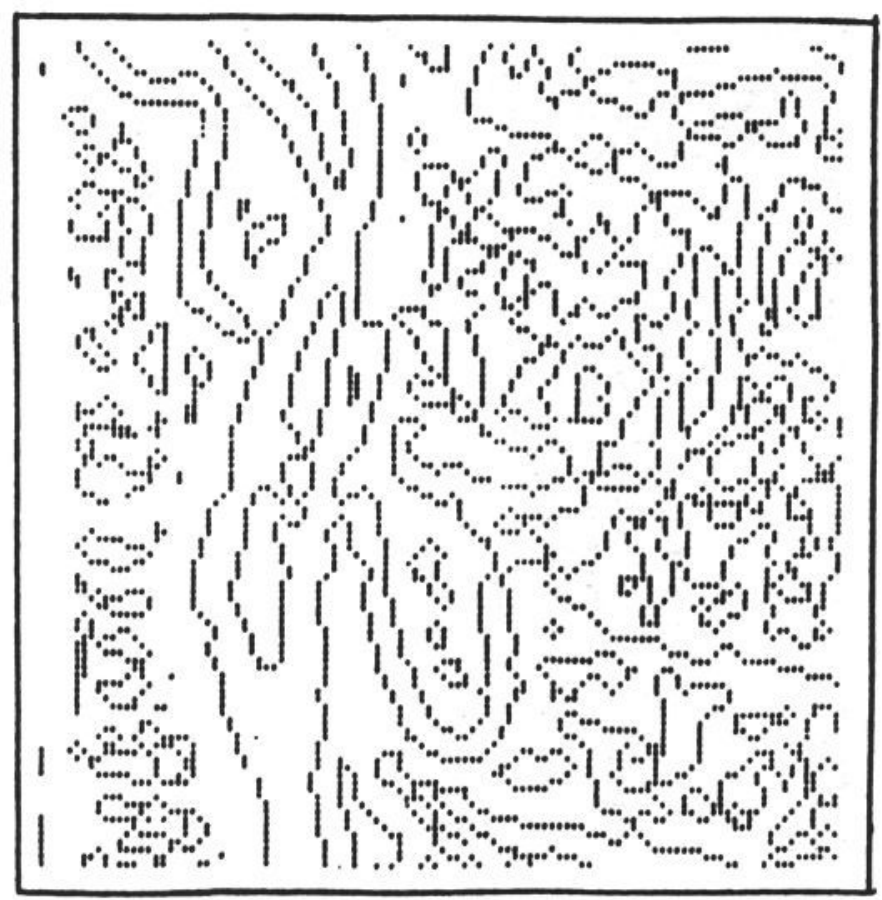

Figure 14 Angio 2: Marr-Hildreth operator output with width $=11$ pixels

convergence. However, unlike Rosenfeld's algorithms, the scale of the elements being linked at each stage is not predetermined, but is set by local data. As with other pyramidal algorithms, scale increases as one ascends from the lowest level to the highest in our data structure, but the level of a feature in the hierarchy specifies the number of generations of subfeatures below it, rather than absolute size.

The different approaches used in the MGP grouping method and conventional edge detectors have made it difficult to decide which stages of the MGP grouping method to include in a comparison with more conventional operators. Objective comparison was also made difficult because of user input to the processing. To obtain optimum results from the Canny and Marr Hildreth operators required several experiments with different scale parameters using expert knowledge to assess the results before the optimum setting for this particular application was established. our preliminary evaluation of the MGP grouping 
method indicates that when applied to angiographic subtraction images it performs similarly to the Canny operator in the detection of edge fragments. A significant advantage of the IIGP grouping method arose in the implementation of later stages of processing. The linking of anti-symmetric edge sections and derivation of the centre-line and width of ridges were all natural extensions of the earlier grouping stages and very successful in operation.

The images so far examined have relatively strong features and we have yet to evaluate the MGP grouping method in very noisy images. Future extensions of the MGP grouping method should help to ensure good performance in noisy images. Firstly, small peaks or ridges due to noise, on a larger "hillside" representing the edge of a vessel, will be categorised as subparts of the larger edge and will not prevent detection and characterisation of the larger structure. Secondly, if local links are not established at any level of the pyramid at any location, then grouping will be attempted over a wider area. This is the situation which will often arise in noisy images. Processing time will be increased, but where strong local structure is found, this time penalty will be avoided.

In many types of image, the shape of the grey-level profiles normal to edges carries important information ${ }^{9}$ which is potentially useful both during image-driven segmentation and during image interpretation using doma in specific knowledge. This is particularly true in quantitative angiography, where many of the calculations about vessel width, cross-sectional area, crosssectional shape and other parameters are derived from the profile of grey level values along a track perpendicular to the long axis of the vessello. Iraditionally the construction of these transverse density profiles has required several initial steps: vessel recognition (usually with extensive user interaction); edge localisation; centre-line localis- ation; calculation of the normal to the centre-line; and selection of nearest pixels for grey level sampling. The MGP grouping method described here provides a much more efficient processing sequence.

There are still many aspects of the MGP grouping method to be explored and evaluated. The preliminary results described here have been sufficiently encouraging to justify further investigation and our work in this area is continuing. Future extensions will include the detection and classification of oth e $r$ topological features such as valleys and grey-level maxima and minima at different scales (hill tops and valley bottoms). In this way the whole image will be represented without using domainspecific knowledge as a hierarchical tree structure which codes the relationship between features at multiple scales.

\section{References}

1. Ritchings $R T$ and Colchester ACF. (1986) Detection of abnormalities on carotid angiograms using syntactic techniques. $\quad \mathrm{P}$ a $\mathrm{t} \mathrm{t}$ e $\mathrm{rn}$ Recognition Letters $\underline{4} 367-$ 374 .

2. Ritchings RT, Colchester ACF and $\mathrm{Wang} \mathrm{H}-\mathrm{Q}$. ( 1986 ) Knowledge based analysis of carotid arteriograms. Image and Vision Computing 3 217-222.

3. Wang $H-Q$, Ritchings RT and Colchester ACF. (1987) An image understanding system for carotid angiograms. Image and Vision Computing $\underline{5}$ 79-84.

4. Pizer SM, Oliver WR, and Bloomberg SH. ( 1987 ) Hierarchical shape description via the multi-resolution symmetric axis transforms. Pattern Analysis \& Machine Intelligence PAMI-9 505-511. 
5. Koenderink JJ (1988) Image structure. In Viergever MA and Todd-Pokropek A (eds) Mathematics and Computer Science in Medical Imaging, Berlin: Springer, 67-104.

6. Shirai Y (1973) A context sensitive line-finder. Artificial Intelligence 4 : 95-119

7. Baugher $S$ and Rosenfeld $A$ (1986) Boundary localisation in an image pyramid. Pattern Recognition 19: 373-395.
8. Rosenfeld A (1987) Pyramid algorithms for efficient vision, TR 1866, Center for Automation Research, Univ. Maryland, College Park, June 1987.

9. Sleigh AC ( 1985$)$ The extraction of boundaries using local measures driven by $\mathrm{rules}$. BPRA $3 \mathrm{rd}$ International Conference, st. Andrew's, Scotland.

10. Colchester ACF (1985) The effect of changing $\mathrm{Pa} \mathrm{CO}_{2}$ on cerebral artery calibre estimated by a new technique of dynamic quantitative digital angiography. PhD Thesis, University of London. 